\title{
Review: targeted, multicomponent programmes, delivered by health care professionals most effective at reducing risk factors for depression
}

Jane-Llopis E, Hosman C, Jenkins R, et al. Predictors of efficacy in depression prevention programmes: meta-analysis. Br J Psychiatry 2003;183:384-97.

What are the population, programme, or research design characteristics that will predict the efficacy of primary prevention programmes designed to reduce risk factors for depression?

\section{METHODS}

$\square$

Design: Systematic review with meta-analysis

Data sources: ERIC, MEDLINE, PsycINFO, and Current Contents,

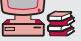
from 1985-2000.

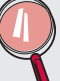

Study selection and analysis: Studies that had either a randomised control, or an equivalent comparison group. Analysis: weighted effect size estimates were determined for each outcome assessed. Study heterogeneity was assessed using the $Q$ statistic.

Outcomes: Prevention of depression; improvement of protective factors for depression (for example, self esteem), or reduction of risk factors for depression (for example, negative thinking).

\section{MAIN RESULTS}

Data were extracted from 69 studies (number of people not stated). The weighted mean effect size was 0.22 (95\% CI 0.14 to 0.30 ). Programme characteristics that significantly improved outcomes include: session lengths of 60-90 minutes (compared with session lengths $<60$ minutes or $>90$ minutes; $\mathrm{p}<0.05$ for both comparisons); provision by healthcare professionals with or without lay personnel (compared with provision by lay personnel alone; $\mathrm{p}<0.01$ for both comparisons); inclusion of competence enhancement techniques (compared with programmes that did not include them; $\mathrm{p}<0.001$ ); and using three or more methods (compared with one or two methods; $p=0.027$ ). Programmes including social support resulted in larger effect sizes for older people only (compared with no social support, $\mathrm{p}<0.001$ ) but were not significantly different in other age groups $(\mathrm{p}=0.55)$.

\section{CONCLUSIONS}

Programmes directed toward reducing risk factors for depression are more effective if they have session lengths of 60-90 minutes, are

For correspondence: Dr E Jane-Llopis, Department of Clinical Psychology and Personality, University of Nijemegen, The Netherlands; llopis@psych.kun.nl Sources of funding: Dutch Health Research and Development Council (ZON). delivered by a healthcare professional, include more than two methods, and include competence enhancement techniques.

\section{Commentary}

he scope of this meta-analysis is broad: gains in terms of improved depression symptoms are shown in whole populations, subpopulations, and in various settings. It opens the field to the scrutiny of mental healthcare providers, educators, and practitioners in other related sectors, and equally important to policy makers.

Preventive psychiatry has been slow to gain credibility for several reasons. Psychiatry has remained outside the public health tradition, whereas experience of disease prevention resides in the field of public health with its fundamental concepts of health and illness as a continuum and as multifactorial in origin. This analysis provides evidence in a form useful to practitioners and policy makers; it assists in creating the virtuous circle of evidence, conviction, innovation, activity, and evaluation. It also underlines the relative scarcity of well evaluated interventions in this area.

Service providers can be encouraged to consider a range of interventions at several levels. For example, practitioners can consider interventions for elderly people in particular settings such as nursing homes, as well as offering expertise to programmes that seek to improve social support for all elderly people in the community. Principles of programme intervention are being defined, but also the diversity of approaches available, and the understanding that programmes need to be designed and evaluated locally.

The finding that programmes including social support for age groups other than older people did generally worse than those that did not is counter intuitive and requires further study. It is one of the important leads that this analysis provides for further programme and policy development and evaluation.

The interventions reviewed are all well evaluated programmes, mostly from the Western world. A challenge now is to evaluate and refine good practice in settings and vulnerable groups other than those already included in this analysis, especially in developing countries. In all settings it is important to extend the available evidence to longer term study of outcomes, and to consider the range of outcomes from any one intervention. Mental health service providers can be ready to be involved in the evaluation and shaping of programmes in physical health such as diabetes and HIV, and addictions, to ensure that the mental health improvement is optimised and evaluated.

Helen E Herrman, MD MBBS

Department of Psychiatry, University of Melbourne and St Vincent's Health Melbourne, Melbourne, Victoria, Australia 